\title{
Gibbs free energy difference between the undercooled liquid and the $\beta$ phase of a Ti-Cr alloy
}

\author{
K. Ohsaka and E. H. Trinh \\ Jet Propulsion Laboratory, California Institute of Technology, Pasadena, California 91109 \\ J. C. Holzer and W. L. Johnson \\ W. M. Keck Laboratory of Engineering Materials, California Institute of Technology, Pasadena, \\ California 91125
}

(Received 22 July 1991; accepted for publication 11 December 1991)

\begin{abstract}
The heat of fusion and the specific heats of the solid and liquid have been experimentally determined for a $\mathrm{Ti}_{60} \mathrm{Cr}_{40}$ alloy. The data are used to evaluate the Gibbs free energy difference, $\Delta G$, between the liquid and the $\beta$ phase as a function of temperature to verify a reported spontaneous vitrification (SV) of the $\beta$ phase in Ti-Cr alloys. The results show that SV of an undistorted $\beta$ phase in the $\mathrm{Ti}_{60} \mathrm{Cr}_{40}$ alloy at $873 \mathrm{~K}$ is not feasible because $\Delta G$ is positive at the temperature. However, $\Delta G$ may become negative with additional excess free energy to the $\beta$ phase in the form of defects.
\end{abstract}

In a series of papers, Blatter, von Allmen and their colleagues have reported the spontaneous vitrification (SV) of Ti-Cr alloys when a metastable $\beta$ phase was annealed at a subeutectoid temperature. ${ }^{1-4}$ The $\beta$ phase (bcc) is a disordered solution which is stable only at high temperatures but can be retained at low temperatures with a moderate quenching rate. Phase decomposition studies ${ }^{5,6}$ and a thermodynamic model ${ }^{5}$ indicate that the quenched $\beta$ phase is within the spinodal lines and is strongly subject to decomposition. However, Blatter et al. found that the $\beta$ phase was transformed into an amorphous phase upon annealing at $873 \mathrm{~K}$ for $6 \mathrm{~h}$. Although the experimental evidence is not totally convincing (to our knowledge, only one independent confirmation has been reported ${ }^{7}$ ), the possibility of SV in Ti-Cr alloys has been sought on the basis of a thermodynamic criterion. ${ }^{1,8,9}$ The thermodynamic criterion dictates that the free energy of the amorphous phase must be lower than that of the $\beta$ phase at the SV temperature. However, the conclusions are not conclusive because the analyses heavily rely on models and assumptions. The Gibbs free energy difference, $\Delta G(T)$, between the liquid and the $\beta$ phase at the temperature, $T$, is formally written as

$$
\begin{aligned}
\Delta G(T)= & \left(\Delta H_{f}+\int_{T_{m}}^{T} \Delta C_{p} d T\right) \\
& -T\left(\Delta S_{f}+\int_{T_{m}}^{T} \Delta C_{p} / T d T\right),
\end{aligned}
$$

where $\Delta H_{f}$ and $\Delta S_{f}$ are the enthalpy and the entropy of fusion, respectively, $T_{m}$ is the melting point (a congruent melting for the present case) and $\Delta C_{p}=C_{p}^{1}-C_{p}^{\beta}$ is the difference in specific heats of two phases. In this letter, we calculate $\Delta G(T)$ using the experimentally determined $\Delta H_{f}, C_{p}^{\beta}$ and $C_{p}^{1}$, and evaluate the feasibility of SV in $\mathrm{Ti}-\mathrm{Cr}$ alloys from a thermodynamic point of view without getting into the kinetic aspect of the transition.

We used a Perkin-Elmer DSC 4, a high temperature SETARAM DSC $2000 \mathrm{~K}$ and a drop calorimeter for calorimetric measurements. A detailed description of the drop calorimeter has been provided elsewhere. ${ }^{10}$ Buttons of the $\mathrm{Ti}_{60} \mathrm{Cr}_{40}$ alloy were prepared by induction melting of 99.7\% pure $\mathrm{Ti}$ and $99.9 \%$ pure $\mathrm{Cr}$ on a silver boat in an $\mathrm{Ar}$ atmosphere. $\mathrm{X}$-ray diffraction showed that the as-prepared alloys were predominated by the metastably retained $\beta$ phase. The diffraction lines were narrow, indicating very little distortion of the lattice. We first determined $T_{m}$ and $\Delta H_{f}$ using the high temperature DSC. The results are 1688 $\pm 10 \mathrm{~K}$ and $15.7 \pm 3.7 \mathrm{~kJ} / \mathrm{mol}$, respectively, which are in good agreement with the assessed phase diagram. ${ }^{11}$

We next measured the enthalpy of the $\mathrm{Ti}_{60} \mathrm{Cr}_{40}$ liquid alloy as a function of temperature using the drop calorimeter. The result is shown in Fig. 1. The enthalpy of the $\beta$ phase at $298 \mathrm{~K}$ is assigned to zero. The enthalpy of the liquid, $H^{l}$, (presented as the solid circles) is fitted with two commonly used empirical equations for the enthalpy by a regression method. The results are

$$
H^{1}(T)=-26.3+5.10 \times 10^{-2} T \quad(\mathrm{~kJ} / \mathrm{mol})
$$

and

$$
H^{1}(T)=9.98+0.0405 T-3.12 \times 10^{4} / T \quad(\mathrm{~kJ} / \mathrm{mol}) .
$$

Equation (3) is drawn in Fig. 1. By definition, $C_{p}^{1}$ is given by

$$
C_{p}^{1}=d H^{1} / d T=51.0 \quad(\mathrm{~J} / \mathrm{mol} \mathrm{K}) \quad[\text { using Eq. (2)] }
$$

and

$$
\begin{aligned}
C_{p}^{1}= & 40.5+3.12 \\
& \times 10^{7} / T^{2} \quad(\mathrm{~J} / \mathrm{mol} \mathrm{K}) \text { [using Eq. (3) ] }
\end{aligned}
$$

For comparison with the specific heat of the $\beta$ phase, Eq. (5) is drawn in Fig. 2.

For the $\beta$ phase, at low temperatures $(<700 \mathrm{~K})$ where the retained $\beta$ phase was stable for the duration of the measurement, we directly measured $C_{p}^{\beta}$ using the DSC 4 . The result is shown in Fig. 2. as the open circles. At high temperatures $(>1400 \mathrm{~K}$ ) where the $\beta$ phase could be retained in the dropped sample, we measured the enthalpy of 


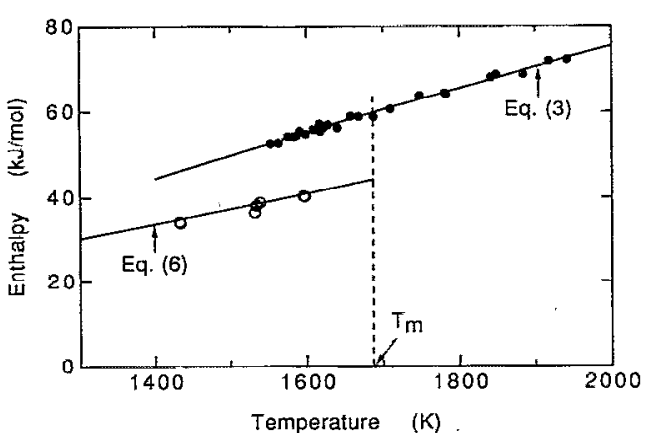

FIG. 1. The enthalpy of the $\mathrm{Ti}_{60} \mathrm{Cr}_{40}$ alloy measured by a drop calorimeter. Equations (3) and (6) are filted equations. Equation ( 6 ) is determined by taking into account the specific heat data shown in Fig. 2 .

the $\beta$ phase, $H^{\beta}$, using the drop calorimeter. The result is shown in Fig. 1 as the open circles. The two sets of data are well fitted simultaneously by

$$
\begin{aligned}
H^{\beta}(T)= & -7.17+0.0227 T+4.59 \\
& \times 10^{-6} T^{2} \quad(\mathrm{~kJ} / \mathrm{mol})
\end{aligned}
$$

and

$$
C_{p}^{\beta}(T)=22.7+9.18 \times 10^{-3} T \quad(\mathrm{~J} / \mathrm{mol} \mathrm{K}) .
$$

Equations (6) and (7) are drawn in Figs. 1 and 2, respectively. The heat of fusion calculated by taking the difference between Eqs. (3) and (6) at $T_{m}$ is $15.5 \mathrm{~kJ} / \mathrm{mol}$, which is in very good agreement with the result from the DSC measurement.

We are now able to calculate $\Delta G$ of the $\mathrm{Ti}_{60} \mathrm{Cr}_{40}$ alloy using the experimentally determined $\Delta C_{p}$ and $\Delta H_{f}$. However, $C_{p}^{1}$ is only determined at the limited undercooling level ( $<135 \mathrm{~K})$. Thus, we assume that Eqs. (4) or (5) can be extrapolated into low temperatures. The extrapolation of Eq. (4) underestimates $\Delta C_{p}$ because $C_{p}^{1}$ of glass-forming alloys generally increases as undercooling increases due to the decrease in configurational entropy of the liquid. ${ }^{12}$ The extrapolation of Eq. (5) is more realistic but may overestimate $\Delta C_{p}$. We also have a fairly large uncertainty in $\Delta H_{f}(12.0-19.0 \mathrm{~kJ} / \mathrm{mol})$ measured by DSC. Considering these uncertainties in $C_{p}^{1}$ and $\Delta H_{f}$, we decided to calculate $\Delta G$ with several combinations of $\Delta C_{p}$ and $\Delta H_{f}$. The results

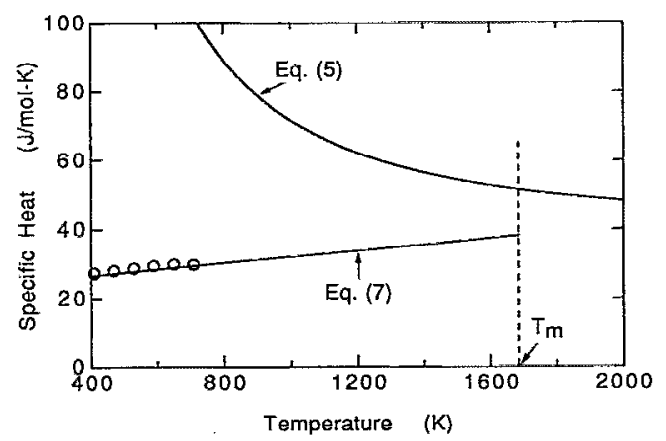

FIG. 2. The specific heats of the $\mathrm{Ti}_{60} \mathrm{Cr}_{40}$ alloy calculated from Eqs. (5) and (7). The open circles are values measured by DSC.

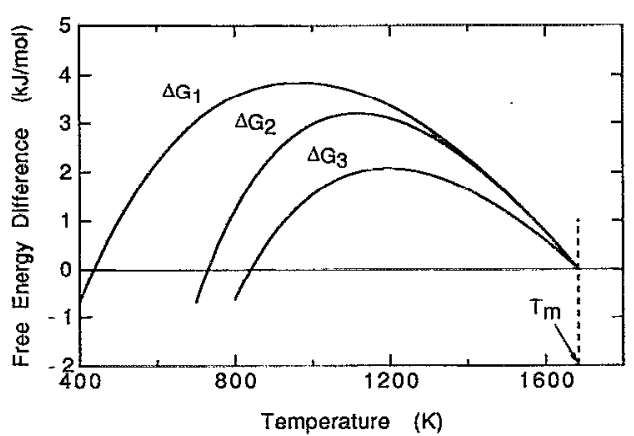

FIG. 3. The free energy difference between the liquid and the $\beta$ phase for the $\mathrm{Ti}_{60} \mathrm{Cr}_{40}$ alloy.

are shown in Fig. 3: $\Delta G_{1}$ is obtained with Eqs. (4) and (7) and $\Delta H_{f}=15.5 \mathrm{~kJ} / \mathrm{mol}$; and $\Delta G_{3}$ is obtained with Eqs. (5) and (7) and $\Delta H_{f}=15.5 \mathrm{~kJ} / \mathrm{mol}$; and $\Delta G_{3}$ is obtained with Eqs. (5) and (7) and $\Delta H_{f}=12.0 \mathrm{~kJ} / \mathrm{mol}$. The temperatures, $T_{c}$, where $\Delta G=0$, are 430,735 , and $840 \mathrm{~K}$ for $\Delta G_{1}$, $\Delta G_{2}$, and $\Delta G_{3}$, respectively.

In Fig. 3, the temperature at which $\Delta G$ reaches a maximum represents the Kauzmann temperature, $T_{K}{ }^{13}$ Below $T_{K}$, the entropy of the undercooled liquid becomes smaller than that of the $\beta$ phase. This situation seems paradoxical but is feasible if we assume that the liquid is topologically ordered and the $\beta$ phase is disordered. Thus, the liquid may be undercooled below $T_{K}$, but is eventually transformed into glass (amorphous phase) at the glass transition temperature, $T_{g}$ (which has not been determined in the present system). The specific heat of glass is generally slightly larger than that of its corresponding crystalline phase; therefore, the $\Delta G$ 's in Fig. 3 are not valid below $T_{g}$. Consequently, if $T_{g}>T_{c}$, then the actual $T_{c}$ becomes much lower than that which is shown in Fig. 3. It is clear that even with the most favorable situation, i.e., $\Delta G_{3}$ with $T_{c}=840 \mathrm{~K}$, the present results do not predict $\mathrm{SV}$ of the undistorted $\beta$ phase in the $\mathrm{Ti}_{60} \mathrm{Cr}_{40}$ alloy at $873 \mathrm{~K}$. We may add that our $\beta$ phase sample annealed at $873 \mathrm{~K}$ for several hours was decomposed into the stable phases.

Additional excess free energy to the $\beta$ phase has been suggested in the form of the interstitial-vacancy pairs ${ }^{14}$ or lattice distortion, ${ }^{9,15}$ which may raise the free energy of the $\beta$ phase above that of glass at $873 \mathrm{~K}$. For instance, an additional vacancy concentration of $10^{-3}$ in the $\beta$ phase is required to nullify $\Delta G_{3}=0.4 \mathrm{~kJ} / \mathrm{mol}$ at $873 \mathrm{~K}$ assuming that the enthalpy for vacancy formation is to the order of $1 \mathrm{eV}$. It has also been suggested that impurities may play a role in SV. ${ }^{16}$ We speculate that if this is the case, the role of the impurities is to provide favorable heterogeneous nucleation sites for the amorphous phase rather than to substantially increase the free energy of the $\beta$ phase.

This work represents one phase of research carried out at the Jet Propulsion Laboratory, California Institute of Technology, under contract with the National Aeronautics and Space Administration (NASA). Two of the authors (J. C. H. and W, L. J.) acknowledge the support of NASA under Contract No. NAS 496954MG3203550. 
${ }^{\prime}$ A. Blatter and M. von Allmen, Phys. Rev. Lett. 54, 2103 (1985).

${ }^{2}$ M. von Allmen and A. Blatter, Appl. Phys. Lett. 50, 1873 (1987).

${ }^{3}$ A. Blatter, M. von Allmen, and N. Baltzcr, J. Appl. Phys. 62, 276 (1987).

${ }^{4}$ Ch. Wirz, A. Blatter, N. Baltzer, and M. von Allmen, Phys. Rev. B 42, 6993 (1990).

${ }^{5}$ E. S. Menon and H. I. Aaronson, Acta Met. 34, 1963 (1986).

${ }^{6}$ Y. Ikematsu, M. Doi, and T. Miyazaki, J. Mater. Sci. 26, 2071 (1991).

${ }^{7}$ Y.-G. Kim and J.-Y. Lee, J. Non-Cryst. Solids 122, 269 (1990).

${ }^{*}$ A. L. Greer, J. Less-Common Met. 140, 327 (1988).
${ }^{9}$ L. J. Gallego, J. A. Somoza, and J. A. Alonso, Physica B 160, 108 (1989).

${ }^{10} \mathrm{~K}$. Ohsaka, J. R. Gatewood, and E. H. Trinh, Scripta Met. Mat. 25, 1459 (1991).

${ }^{11}$ J. L. Murray, Bull. Alloy Phase Diag. 2, 174 (1981).

${ }^{12}$ H. S. Chen and D. Turnbull, J. Appl. Phys. 38, 3646 (1967).

${ }^{13}$ W. Kauzmann, Chem. Rev. 43, 219 (1948).

${ }^{14}$ W. L. Johnson, Prog. Mater. Sci. 30, 81 (1986).

${ }^{15}$ A. Blatter, U. Kambli, Ch. Wiez, R. Giovanoli, K. Dyrbye, and J. Bottiger, Phys. Rev. B 40, 12503 (1989).

${ }^{16}$ W. Sinkler and D. E. Luzzi, Mater. Soc. Symp. Proc. (to be published). 